\title{
Dental Implants Surface in vitro Decontamination Protocols
}

\author{
Vanessa Coelho Batalha ${ }^{1}$ Raquel Abreu Bueno ${ }^{1}$ Edemar Fronchetti Junior ${ }^{1}$ \\ José Ricardo Mariano ${ }^{2}$ Gabriela Cristina Santin ${ }^{3}$ Karina Maria Salvatore Freitas ${ }^{4, \odot}$ \\ Mariana Aparecida Lopes Ortiz ${ }^{5}$ Samira Salmeron ${ }^{1}$
}

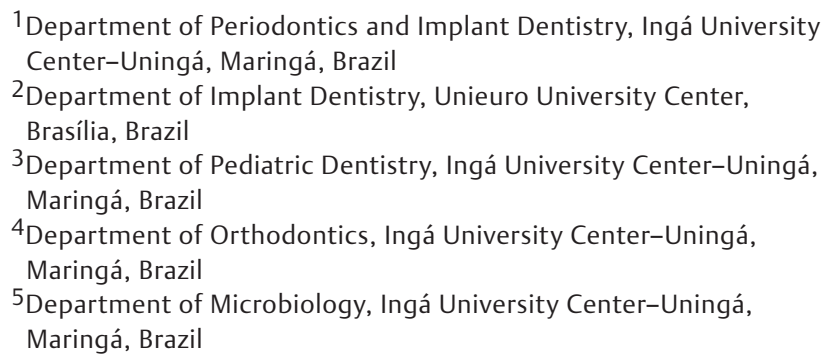

Address for correspondence Samira Salmeron, DDS, MSc, PhD, Department of Periodontics and Implant Dentistry, Ingá University Center-Uningá, Rod PR 317, Zip code 87035-510, Maringá, Brazil (e-mail: salmeronsamira@gmail.com).

Eur J Dent 2021;15:407-411

\section{Abstract}

\section{Keywords}

- peri-implantitis

- sodium bicarbonate

- photodynamic therapy

- dental implants

- decontamination
Objective The number of patients rehabilitated with dental implants has contributed to increased incidence of peri-implant diseases. Due to complex and difficult treatment, peri-implantitis is a challenge and an efficient clinical protocol is not yet established. Aim of this study was to evaluate the efficacy of two protocols for in vitro decontamination of dental implants surface.

Materials and Methods Twenty titanium implants (BioHE-Bioconect) were used. Implants were divided into five groups $(n=4)$. NC group (negative control): sterile implants; PC group (positive control): biofilm contaminated implants; $S$ group: biofilm contaminated implants, brushed with sterile saline; SB group: biofilm contaminated implants, brushed with sterile saline and treated with air-powder abrasive system with sodium bicarbonate ( 1 minute); and antimicrobial photodynamic therapy (aPDT) group: biofilm contaminated implants, brushed with sterile saline and treated with antimicrobial photodynamic therapy (red laser + toluidine blue $\mathrm{O}$ ). The implants were contaminated in vitro with subgingival biofilm and distributed in groups PC, S, SB, and aPDT. Each group received the respective decontamination treatment, except groups NC and PC. Then, all implants were placed in tubes containing culture medium for later sowing and counting of colony-forming units (CFUs).

Statistical Analysis One-way analysis of variance and Tukey tests were performed, at $5 \%$ significance level.

Results Significantly fewer CFUs were observed in the aPDT group $\left(19.38 \times 10^{5}\right)$ when compared with groups SB $\left(26.88 \times 10^{5}\right), S\left(47.75 \times 10^{5}\right)$, and PC $\left(59.88 \times 10^{5}\right)(p<0.01)$. Both the aPDT and SB groups were statistically different from the NC group $(p<0.01)$. Conclusion Proposed protocols, using air-powder abrasive system with sodium bicarbonate and aPDT, showed to be efficacious in the decontamination of dental implants surface in vitro.
DOI https://doi.org/ $10.1055 / \mathrm{s}-0040-1721550$ ISSN 1305-7456.
(C) 2020. European Journal of Dentistry.

This is an open access article published by Thieme under the terms of the Creative Commons Attribution-NonDerivative-NonCommercial-License, permitting copying and reproduction so long as the original work is given appropriate credit. Contents may not be used for commercial purposes, or adapted, remixed, transformed or built upon. (https://creativecommons.org/licenses/by-nc-nd/4.0/).

Thieme Medical and Scientific Publishers Pvt. Ltd. A-12, 2nd Floor, Sector 2, Noida-201301 UP, India 


\section{Introduction}

Osseointegrated dental implants are well established as a predictable treatment option, with high success ${ }^{1}$ and survival rates. ${ }^{2}$ However, failures can occur and, with increased number of implants installed, an increase in related complications is also expected. ${ }^{3}$

Peri-implant diseases are among the main complications, with incidence and prevalence rates following the increase in dental implant installation. ${ }^{4}$ Recently, peri-implant diseases were classified into peri-implant mucositis and peri-implantitis ${ }^{5}$ and present the biofilm as the main etiologic agent. ${ }^{5,6}$ Peri-implantitis is considered a clinically complex and irreversible condition, ${ }^{4,7}$ and is characterized by inflammation of the soft tissue around the implant with subsequent and progressive bone loss. ${ }^{8}$

The management of peri-implantitis can vary according to disease severity and extent, and nonsurgical or surgical approaches are indicated depending on the clinical and radiographic findings. ${ }^{9}$ Nonsurgical therapy includes the use of mechanical methods that promotes biofilm removal by using curettes, ultrasonic scalers, air abrasive systems, and titanium brushes. ${ }^{9}$ Chemical methods like citric acid and lasers, besides antiseptics and antibiotics ${ }^{9}$ are suggested to be used in association with mechanical methods. Although nonsurgical therapy is an option, studies have shown that surgical treatment is recommended for peri-implantitis to achieve more favorable outcomes. ${ }^{9}$

One of the main difficulties in the peri-implantitis treatment is the implant surface decontamination, which seems to be a major concern ${ }^{7,10-14}$ in the process that aims at reosseointegration. ${ }^{15,16}$ Thus, several methods of implant surface decontamination have been proposed, both mechanical and chemical, in surgical access. ${ }^{10,15,17}$ However, to date, no protocol has been established as the gold standard for peri-implantitis treatment. ${ }^{15,18,19}$

The literature shows that best decontamination results can be achieved when both mechanical and chemical methods are used., ${ }^{9,20,21}$ As a mechanical method, sandblasting with abrasive particles has been used to treat peri-implantitis with positive effects. ${ }^{16,22,23}$ The sandblasting systems use abrasive powder introduced into a stream of compressed air, and these powder particles gain kinetic energy from a flow of water and compressed air. ${ }^{24}$ So, powder is an important parameter in the sandblasting effectiveness. ${ }^{16}$ Among the available types, sodium bicarbonate proved to be very effective in removing bacteria from implant surfaces. ${ }^{16}$ Regarding peri-implantitis treatment, studies show positive results with air-powder abrasive system with sodium bicarbonate using different time protocols from 60 seconds to 2 minutes..$^{25-27}$ Disadvantages as implant surface changes are also presented. ${ }^{16,25}$ Thus, concerns about the time and efficacy are still raised in the literature, as well as changes in implant surface topography.

In the last decades, lasers associated with photosensitizing agents, in antimicrobial photodynamic therapy (aPDT), have been used as a chemical method for dental implant surface decontamination with promising results. ${ }^{19,28}$ The aPDT is a minimally invasive, nontoxic, and safe method ${ }^{14}$ based on the use of a photosensitizer, usually a dye activated by light with a specific wavelength, which can kill bacteria by generating highly reactive oxygen species. ${ }^{14,29}$ The anti-infective treatment with aPDT uses a low-level laser after application of photosensitizing agents like toluidine blue and methylene blue or indocyanine green, ${ }^{13,30}$ the only three photosensitizers clinically approved for use in humans in combination with light. ${ }^{30,31}$ Recent studies have shown that aPDT seems to be effective in reducing bacterial load in peri-implantitis and is a potential alternative therapy. ${ }^{14}$ However, some studies showed no difference in the effects of aPDT when compared with conventional therapies in peri-implantitis clinical treatment. ${ }^{32}$ Thus, despite the promising results of aPDT, there is no consensus and a great diversity of protocols in related parameters, and an ideal protocol is not yet established.

The present study aims to contribute to the advancement of knowledge on peri-implantitis treatment, evaluating the efficacy of two protocols, mechanical and chemical, for in vitro decontamination of metallic implants surface.

\section{Materials and Methods}

This study was approved by the research ethics committee of the Inga University Center (number 3.072.210) regarding the biofilm collection. A sample size calculation was performed, and the result showed that 20 implants were necessary for this study. Therefore, 20 grade IV commercially available pure titanium implants were used, with a surface treated with double acid etching, cylindrical, external hexagon, measuring $5 \mathrm{~mm}$ in diameter and $18 \mathrm{~mm}$ in length (BioHE-Bioconect; Itapira, Brazil), sterilized from the factory. The dental implants were randomly assigned to the following groups $(n=4)$ :

- NC group (negative control): sterile implants.

- PC group (positive control): biofilm contaminated implants.

- S group: biofilm contaminated implants, brushed with sterile saline.

- SB group: biofilm contaminated implants, brushed with sterile saline and treated with air-powder abrasive system with sodium bicarbonate ( 1 minute).

- aPDT group: biofilm contaminated implants, brushed with sterile saline and treated with aPDT.

The implants were contaminated with biofilm, except for the NC group. After signing the informed consent form, a subgingival biofilm sample was collected from a volunteer, diagnosed with severe periodontitis who underwent periodontal treatment at the Ingá University Center. The collection was performed using proper Gracey curettes (HuFriedy Mfg. Co.; Chicago, United States). The selection of the volunteer followed the inclusion criteria: adult subject ( $>18$ years), absence of systemic problems, and nonsmoker. Exclusion criteria were pregnancy, breastfeeding, and use of antibiotics in the last 6 months.

After collection, the biofilm was cultured in sterile brain heart infusion (BHI) broth (Kasvi, São José dos Pinhais, Brazil) 
and gram morphotinturial analysis was performed. The implants were then distributed in groups PC, S, SB, and aPDT, and contaminated in test tubes containing $10 \mathrm{~mL}$ of BHI broth medium and subgingival biofilm, and maintained for 7 days, in a $37^{\circ} \mathrm{C}$ oven, for the formation of biofilm on the implant surfaces. After the in vitro contamination, the implants received the respective treatments, except for the PC implants that did not receive any decontamination treatment. Throughout the process, sterile forceps and gloves were used to avoid contamination of the implants with bacteria other than the biofilm.

Implants from the S, SB, and aPDT groups were brushed with a soft bristle toothbrush (Dentalclean; Londrina, Brazil) with $20 \mathrm{~mL}$ of sterile saline (Eurofarma; São Paulo, Brazil). Twenty brush strokes were performed, covering all implant surfaces (-Fig. 1). After brushing, the SB group implants were treated with high-pressure air-powder abrasive system (Practical Jet-Kondentech; São Carlos, Brazil) with extra-fine granulation sodium bicarbonate (Profhylaxis-Formaden; São José dos Pinhais, Brazil), for 1 minute (-Fig. 2) and rinsed with $10 \mathrm{~mL}$ of sterile saline. The aPDT group implants, after brushing, were placed in 12-well acrylic plates for $1 \mathrm{~min}$ ute, immersed in $3 \mathrm{~mL}$ of toluidine blue $\mathrm{O}$ (Sigma-Aldrich; São Paulo, Brazil) at a concentration of $100 \mu \mathrm{g} / \mathrm{mL}$ diluted in distilled water ${ }^{19}$ ( -Fig. 3). Subsequently, implants were

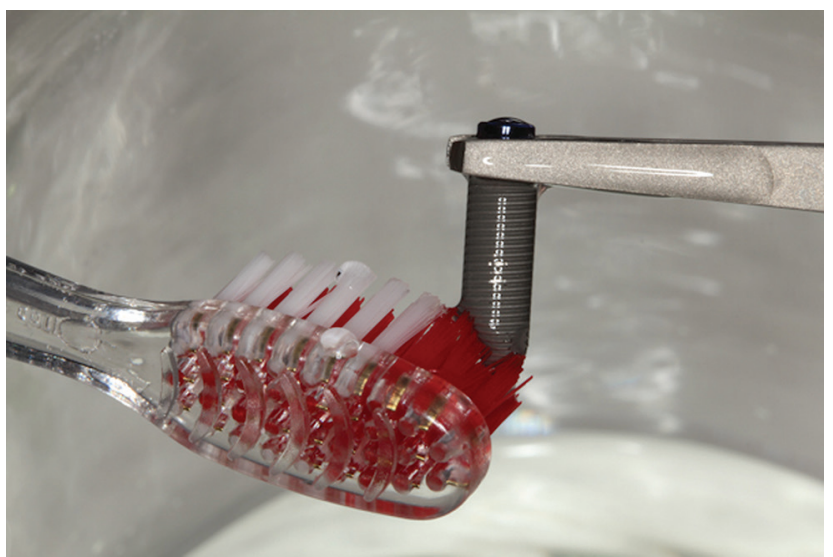

Fig. 1 Dental implant brushed with a soft bristle toothbrush and sterile saline.

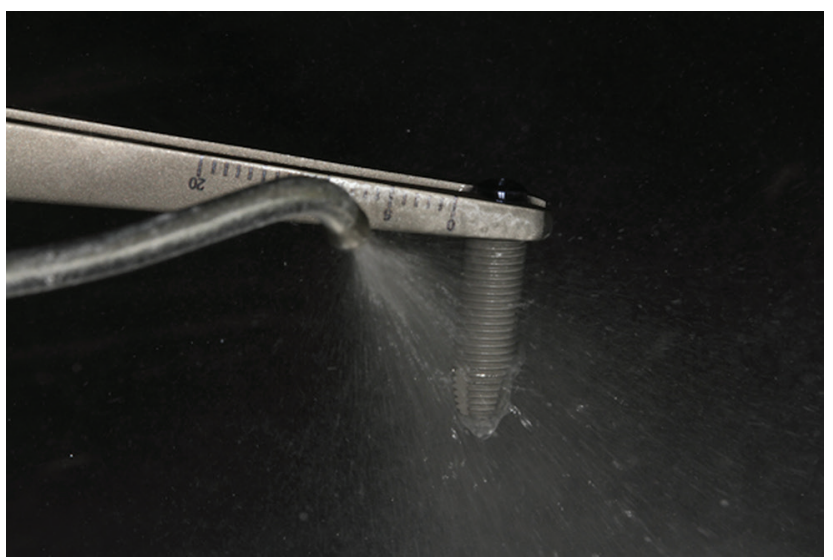

Fig. 2 Dental implant receiving air-powder abrasive system with sodium bicarbonate for 1 minute. irradiated in scan mode with Whitening Lase II (DMC; São Carlos, Brazil) with $600 \mu \mathrm{m}$ diameter fiber optics at a distance of $5 \mathrm{~mm}$ of the surface and divided into four faces (buccal, lingual, mesial, and distal), according to the adapted protocol ${ }^{19}$ (660 nm; $30 \mathrm{~mW} ; 50 \mathrm{~J} / \mathrm{cm}^{2} ; 47$ seconds) (-Fig. 4), and rinsed with $10 \mathrm{~mL}$ of sterile saline.

After decontamination treatments were performed, all implants were inserted in $10 \mathrm{~mL}$ of sterile BHI broth medium. After 24 hours incubated at $37^{\circ} \mathrm{C}$, dilutions and sowing acrylic plates were done in duplicate. These plates were stored in a $\mathrm{CO}_{2}$ anaerobic jar, guaranteeing a condition of microaerophilia, and kept for 48 hours incubated at $37^{\circ} \mathrm{C}$, allowing colonies to grow. Then, the colony-forming units (CFUs) were counted, with the naked eye, by an experienced examiner. The intergroup comparison of CFUs was performed by one-way analysis of variance and Tukey tests, with a significance level of $5 \%$.

\section{Results}

The aPDT group presented the lowest number of CFUs (19.38 $\times$ $\left.10^{5} \pm 1.493\right)$ when compared with the SB group $\left(26.88 \times 10^{5} \pm\right.$ 2.496), S group ( $\left.47.75 \times 10^{5} \pm 4.735\right)$, and the PC group ( 59.88 $\left.\times 10^{5} \pm 1.436\right)$, with statistically significant differences $(p<$ 0.01 ). The SB group had a significantly lower number of CFUs when compared with groups S and PC $(p<0.01)$ and all the

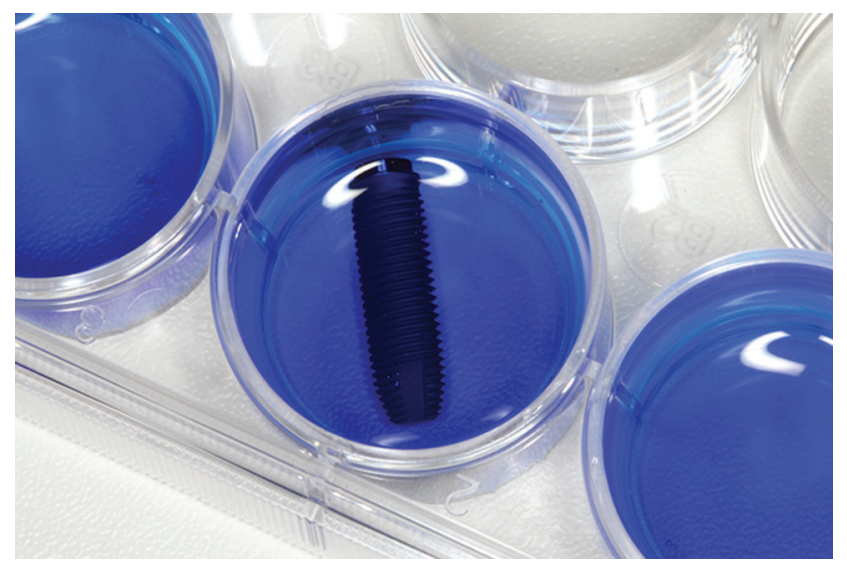

Fig. 3 Dental implant immersed in toluidine blue O (TBO) $(100 \mu \mathrm{g} / \mathrm{mL})$ for 1 minute before irradiation.

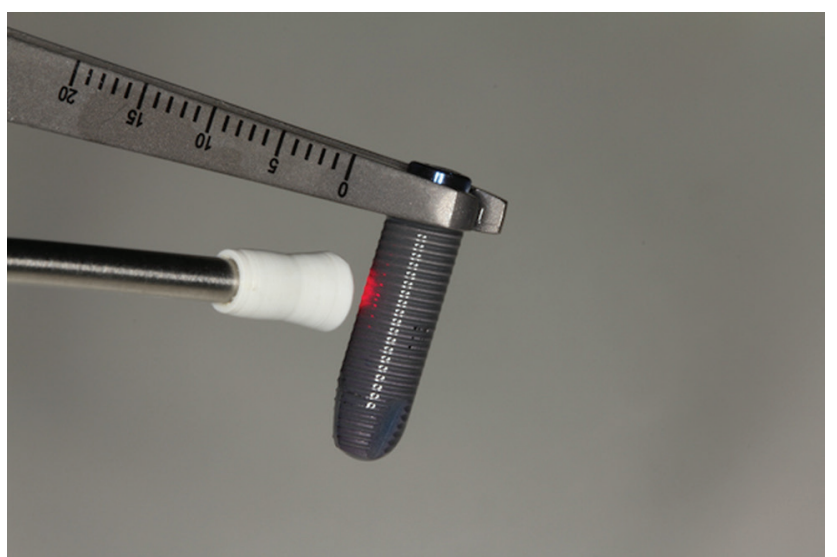

Fig. 4 Implant being irradiated with red laser (Whitening Lase IIDMC, São Carlos, Brazil) for 47 seconds. 
Table 1 Mean values and standard deviation of colonyforming units (CFUs) according to control and test groups

\begin{tabular}{|l|l|}
\hline Experimental groups & Mean \pm SD \\
\hline Negative control & $0 \pm 0^{\mathrm{a}}$ \\
\hline Positive control & $59.88 \times 10^{6} \pm 1.44^{\mathrm{b}}$ \\
\hline Serum & $47.75 \times 10^{6} \pm 4.74^{\mathrm{c}}$ \\
\hline $\begin{array}{l}\text { Air-powder abrasive system with sodium } \\
\text { bicarbonate }\end{array}$ & $26.88 \times 10^{6} \pm 1.49^{\mathrm{d}}$ \\
\hline Antimicrobial photodynamic therapy & $19.38 \times 10^{6} \pm 2.50^{\mathrm{e}}$ \\
\hline
\end{tabular}

Abbreviation: SD, standard deviation.

Note: Different letters indicate significant difference between groups.

groups were statistically different from the NC group, which was not contaminated $(p<0.01)$. The aPDT group, even showing greater efficacy, was not able to completely eliminate the microbial biofilm from the implant surface (-Table $\mathbf{1}$ ).

\section{Discussion}

The air-powder abrasive system with sodium bicarbonate and aPDT, within the conditions presented in this in vitro study, were efficacious in decontaminating surface of dental implants, but did not completely eliminate the microorganisms.

aPDT showed a greater reduction in the number of CFUs when compared with the air-powder abrasive system with sodium bicarbonate. The protocol used was adapted from Salmeron et $\mathrm{al}^{19}$ who also obtained positive results in decontaminating the titanium surface with aPDT. Although these results were significant, the study by Salmeron et $\mathrm{al}^{19}$ was made with titanium discs. Thus, it was decided to use a similar protocol (adapted for use in newer equipment) only in dental implants, to verify the decontaminating potential in this surface conformation, with the design of a real implant and the difficulty of threads decontamination in clinical practice.

One of the biggest challenges and fundamental phases in peri-implantitis treatment is implant surface decontamination. ${ }^{710-14}$ The search for more effective and clinically applicable decontaminating methods continues. In this sense, biofilm and its characteristics become extremely relevant in studies on the topic. ${ }^{33}$ The similarities found between the microbiota of periodontal and peri-implant diseases ${ }^{33-35}$ validated the use of the biofilm in this experimental model. The morphotinturial analysis, performed right after the collection, revealed the presence of Gram-positive and Gram-negative microorganisms, however, there was no biofilm characterization with identification of microorganisms because of the need for more complex analyses that demand higher cost and specific equipment.

Among the mechanical methods available for implant surface decontamination, the air-powder abrasive system with sodium bicarbonate was selected for this study because it is widely used in general clinical routine. The literature brings a wide variety of decontamination protocols ${ }^{25-27}$ and some studies report possible changes in titanium surface topography ${ }^{16,25}$ and even the risk of emphysema. ${ }^{36}$ The present study did not evaluate these possible adverse effects of the air-powder abrasive system with sodium bicarbonate, but its decontamination potential is an easily reproducible in vitro model with similar characteristics to peri-implantitis cases.

The results presented here demonstrate that the air-powder abrasive system with sodium bicarbonate was efficacious, corroborating the results presented in the literature. ${ }^{16,25-27}$ The advantage of this protocol is the short application time, ease of equipment use, and abrasive particles easily found in dental offices.

Combining chemical and mechanical methods for implant surface decontamination can be an interesting alternative as per the results of the present study and the current literature. ${ }^{12,20,21}$ Furthermore, the fact that the $S$ group also presented a decontaminating effect in this study corroborates this hypothesis, suggesting the association of mechanical and chemical methods as an alternative for surface decontamination in cases of peri-implantitis.

\section{Conclusion}

According to the results obtained in this study and considering the limitations of the proposed model, it was possible to conclude that the proposed protocols using air-powder abrasive system with sodium bicarbonate and aPDT demonstrated good efficacy in the decontamination of dental implant surfaces in vitro, but the ideal clinical application protocol is still under study.

\section{Conflict of Interest \\ None declared.}

\section{References}

1 Quirynen M, Herrera D, Teughels W, Sanz M. Implant therapy: 40 years of experience. Periodontol 2000 2014;66(1):7-12

2 Pjetursson BE, Asgeirsson AG,Zwahlen M,Sailer I. Improvements in implant dentistry over the last decade: comparison of survival and complication rates in older and newer publications. Int J Oral Maxillofac Implants 2014;29(Suppl):308-324

3 Dreyer H, Grischke J, Tiede C, et al. Epidemiology and risk factors of peri-implantitis: a systematic review. J Periodontal Res 2018;53(5):657-681

4 Lee CT, Huang YW, Zhu L, Weltman R. Prevalences of periimplantitis and peri-implant mucositis: systematic review and meta-analysis. J Dent 2017;62:1-12

5 Caton JG, Armitage G, Berglundh T, et al. A new classification scheme for periodontal and peri-implant diseases and conditions - introduction and key changes from the 1999 classification. J Periodontol 2018;89(Suppl 1):S1-S8

6 Berglundh T, Armitage G, Araujo MG, et al. Peri-implant diseases and conditions: consensus report of workgroup 4 of the 2017 World Workshop on the Classification of Periodontal and Peri-Implant Diseases and Conditions. J Periodontol 2018;89(Suppl 1):S313-S318

7 Berglundh T, Jepsen S, Stadlinger B, Terheyden H. Periimplantitis and its prevention. Clin Oral Implants Res 2019; 30(2):150-155

8 Schwarz F, Derks J, Monje A, Wang HL. Peri-implantitis. J Periodontol 2018;89(Suppl 1):S267-S290 
9 Rokaya D, Srimaneepong V, Wisitrasameewon W, Humagain M, Thunyakitpisal P. Peri-implantitis update: risk indicators, diagnosis, and treatment. Eur J Dent 2020;14(4):672-682

10 Figuero E, Graziani F, Sanz I, Herrera D, Sanz M. Management of peri-implant mucositis and peri-implantitis. Periodontol 2000 2014;66(1):255-273

11 Matsubara VH, Leong BW, Leong MJL, Lawrence Z, Becker T, Quaranta A. Cleaning potential of different air abrasive powders and their impact on implant surface roughness. Clin Implant Dent Relat Res 2020;22(1):96-104

12 Ramanauskaite A, Daugela P, Juodzbalys G. Treatment of peri-implantitis: meta-analysis of findings in a systematic literature review and novel protocol proposal. Quintessence Int 2016;47(5):379-393

13 Shibli JA. Is laser the best choice for the treatment of peri-implantitis? Photomed Laser Surg 2018;36(11):569-570

14 Fraga RS, Antunes LAA, Fontes KBFDC, Küchler EC, Iorio NLPP, Antunes LS. Is antimicrobial photodynamic therapy effective for microbial load reduction in peri-implantitis treatment? A systematic review and meta-analysis. Photochem Photobiol 2018;94(4):752-759

15 Koo KT, Khoury F, Keeve PL, et al. Implant surface decontamination by surgical treatment of periimplantitis: a literature review. Implant Dent 2019;28(2):173-176

16 Louropoulou A, Slot DE, Van der Weijden F. The effects of mechanical instruments on contaminated titanium dental implant surfaces: a systematic review. Clin Oral Implants Res 2014;25(10):1149-1160

17 Moharrami M, Perrotti V, Iaculli F, Love RM, Quaranta A. Effects of air abrasive decontamination on titanium surfaces: a systematic review of in vitro studies. Clin Implant Dent Relat Res 2019;21(2):398-421

18 Kotsovilis S, Karoussis IK, Trianti M, Fourmousis I. Therapy of peri-implantitis: a systematic review. J Clin Periodontol 2008;35(7):621-629

19 Salmeron S, Rezende ML, Consolaro A, et al. Laser therapy as an effective method for implant surface decontamination: a histomorphometric study in rats. J Periodontol 2013;84(5):641-649

20 Renvert S, Polyzois I. Treatment of pathologic peri-implant pockets. Periodontol 2000 2018;76(1):180-190

21 Sivaramakrishnan G, Sridharan K. Photodynamic therapy for the treatment of peri-implant diseases: a network meta-analysis of randomized controlled trials. Photodiagn Photodyn Ther 2018;21:1-9

22 John G, Becker J, Schwarz F. Effectivity of air-abrasive powder based on glycine and tricalcium phosphate in removal of initial biofilm on titanium and zirconium oxide surfaces in an ex vivo model. Clin Oral Investig 2016;20(4):711-719
23 Tastepe CS, Liu Y, Visscher CM, Wismeijer D. Cleaning and modification of intraorally contaminated titanium discs with calcium phosphate powder abrasive treatment. Clin Oral Implants Res 2013;24(11):1238-1246

24 Moëne R, Décaillet F, Andersen E, Mombelli A. Subgingival plaque removal using a new air-polishing device. J Periodontol 2010;81(1):79-88

25 Wei MCT, Tran C, Meredith N, Walsh LJ. Effectiveness of implant surface debridement using particle beams at differing air pressures. Clin Exp Dent Res 2017;3(4):148-153

26 Quintero DG, Taylor RB, Miller MB, Merchant KR, Pasieta SA. Air-abrasive disinfection of implant surfaces in a simulated model of periimplantitis. Implant Dent 2017;26(3):423-428

27 Deppe H, Horch HH, Henke J, Donath K. Peri-implant care of ailing implants with the carbon dioxide laser. Int J Oral Maxillofac Implants 2001;16(5):659-667

28 Cai Z, Li Y, Wang Y, et al. Antimicrobial effects of photodynamic therapy with antiseptics on Staphylococcus aureus biofilm on titanium surface. Photodiagn Photodyn Ther 2019;25:382-388

29 Hayek RR, Araújo NS, Gioso MA, et al. Comparative study between the effects of photodynamic therapy and conventional therapy on microbial reduction in ligature-induced peri-implantitis in dogs. J Periodontol 2005;76(8):1275-1281

30 Cieplik F, Deng D, Crielaard W, et al. Antimicrobial photodynamic therapy - what we know and what we don't. Crit Rev Microbiol 2018;44(5):571-589

31 Wainwright M, Maisch T, Nonell S, etal. Photoantimicrobials-are we afraid of the light? Lancet Infect Dis 2017;17(2):e49-e55

32 Chambrone L, Wang HL, Romanos GE. Antimicrobial photodynamic therapy for the treatment of periodontitis and peri-implantitis: an American Academy of Periodontology best evidence review. J Periodontol 2018;89(7):783-803

33 Sahrmann P, Gilli F, Wiedemeier DB, Attin T, Schmidlin PR, Karygianni L. The microbiome of peri-implantitis: a systematic review and meta-analysis. Microorganisms 2020;8(5):8

34 Pérez-Chaparro PJ, Duarte PM, ShibliJA, et al. The current weight of evidence of the microbiologic profile associated with periimplantitis: a systematic review. J Periodontol 2016;87(11): 1295-1304

35 Retamal-Valdes B, Formiga MC, Almeida ML, et al. Does subgingival bacterial colonization differ between implants and teeth? A systematic review. Braz Oral Res 2019;33(suppl 1):e064

36 Lee ST, Subu MG, Kwon TG. Emphysema following air-powder abrasive treatment for peri-implantitis. Maxillofac Plast Reconstr Surg 2018;40(1):12 